\title{
Mobile Device Sensing System for Urban Goods Distribution Logistics
}

\author{
Joao C. Ferreira \\ ISTAR-IUL \\ Instituto Universitário de Lisboa (ISCTE-IUL) \\ Lisbon, Portugal \\ Joao.carlos.ferreira@iscte.pt \\ Ana Lucia Martins \\ Business Research Unit (BRU-IUL) \\ Instituto Universitário de Lisboa (ISCTE-IUL) \\ Lisbon, Portugal \\ almartins@iscte.pt
}

\begin{abstract}
This paper presents a low cost mobile application (app) integrated on an Internet of Things (IoT) ecosystem, which uses varied sensor information collected by mobile devices to track and assist on the logistics of urban goods distribution processes. The proposed approach is leveraged by the trend of decreasing costs for mobile data communication in urban environments. Taking into account basic sensor data available in mobile devices (e.g., GPS, accelerometer and magnetometer), it is possible to track the users' movements and adopted routes, identify transit times and driving styles, identify the quality of roads, and track the process of loading/unloading of urban goods. This data can also be analyzed through a data mining process to identify patterns, present driving advice and perform a resource optimization process.
\end{abstract}

Keywords-Mobile device sensing; urban logistics; goods distribution; geographic information system; mobile application.

\section{INTRODUCTION}

According to Reeves [1], the emerging area of ubiquitous computing presents some evident possibilities that appear attractive to various stakeholders in public and private sectors, which will shape the society. It includes enabling technologies available nowadays and in the near future, which can be potentially embedded everywhere, leading to a diversity of different services and applications.

Sensors play an important role in this evolution, and they are also a crucial component of the IoT paradigm and of intelligent monitoring and control systems. Fig. 1 describes our approach, which is based on mobile device sensor data acquisition and subsequent transmission to a central server. This data, which has mainly a continuous range (see the example at Fig. 3), is transformed into discrete classes. Afterwards, with appropriate manipulation, knowledge can be extracted. Sensors available in mobile devices allow seamless data collection, and since users always carry the devices with them, this information can be associated with mobility patterns [2].

978-1-5090-5847-1/17/\$31.00 2017 IEEE

\author{
Vitor Monteiro, Joao L. Afonso \\ Centro Algoritmi \\ University of Minho, Campus of Azurem \\ Guimarães, Portugal \\ \{vmonteiro,jla\}@dei.uminho.pt \\ Jose A. Afonso \\ CMEMS-UMinho Center \\ University of Minho, Campus of Azurem \\ Guimarães, Portugal \\ jose.afonso@dei.uminho.pt
}

Business and logistics processes are quickly adopting mobile applications (apps) to access real-time information, mainly to the optimization of routes and consequently to improve the obtained results. In today's global market, logistics is a complex operation. It demands detailed coordination and approaches to support its processes with smart Information and Communications Technology (ICT) solutions that optimize the distribution of both incoming and outgoing goods within the business, in the distribution centers and on the road.

IoT has great potential for measurement and control logistics operations. The benefits extend across the entire logistics value chain, including internal logistics, warehousing operations, transportation, and last-mile delivery. They have impact on areas such as operational efficiency, safety and security, track and trace, customer experience, and new business models. IoT allows real-time awareness of the distribution process, providing detailed shipment tracking information to end-consumers, ongoing optimization and efficient usage of the available resources. These approaches are enabled through the rise of mobile computing, consumerization of ICT, 5G networks, and big data (BD) analytics, as well as an increasing demand for IoT-based solutions from consumers. Once combined, these factors are enabling logistics providers to adopt IoT at an accelerating rate. This approach can be very helpful in urban logistics, with the creation of associated services for last mile optimization and operational follow-up.

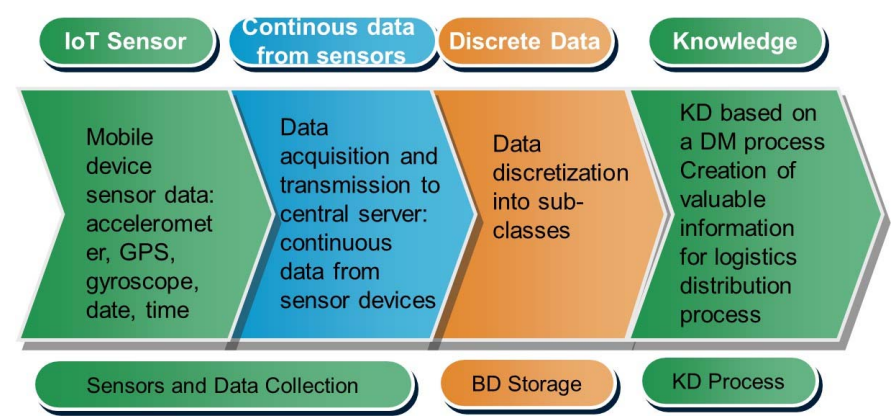

Fig.1. Approach for mobile device sensor data processing. 
Cheap track of goods can be performed, for example, by:

- Low cost Bluetooth Low Energy (BLE) beacons placed inside the goods package, where the signal is captured by mobile devices and interpreted based on previous defined information.

- $\mathrm{QR}$ codes outside of the packages, which may be scanned by a mobile device app.

With this process we track the goods and trigger alerts that can be directed to the recipient's phone via cellular networks.

Companies looking to leverage IoT in their operations should not just consider implementing a single use case within warehousing, transportation, or last-mile delivery. The key to success lies in understanding the convergence of these use cases with each other. Successful implementation of IoT in logistics will require strong collaboration between partners within the supply chain, and the willingness to invest.

\section{SENSORS AND DATA COLLECTION}

Nowadays, most mobile devices integrate the following categories of sensors:

- Motion, location and orientation sensors - These sensors are used to measure the acceleration, rotation or orientation along up to three orthogonal axes, or to provide the position of the user. This category includes: 1) Accelerometers, which measure the combined acceleration due to gravity and body movement along its axes; 2) Gyroscopes, which measure rotational motion along its axes; 3) Magnetometers, which detects the components of the Earth's magnetic field along its axes; and 4) Global Navigation Satellite System (GNSS) receivers, such as Global Positioning System (GPS), which use information received from satellites to infer the device's position.

- Environmental sensors - These sensors are used to measure various environmental parameters, such as ambient temperature, light, pressure and humidity. This category includes thermometers, photodetectors and barometers.

The raw data acquired from the sensors is processed by specific algorithms in order to extract relevant information (e.g., [3, 4, 5]). The main idea and associated processes are illustrated in Fig. 1, where mobile sensor devices can be managed by a proper Software Development Kit (SDK) to extract data.

The first process of the proposed methodology, identified as Process 1: Data Collection in Fig. 2, is responsible for the collection of massive data (big data). This process varies from case to case.

Mobile sensors like accelerometer, GPS and magnetometer are easily accessed through standards functions, such as the ones provided by the Android SDK [11], which includes an emulator of a runtime environment for testing and debugging, as well as classes and interfaces of the Android Sensor Framework in Java language. The sensor framework is part of the Android hardware package. After the sensor data acquisition, proper data manipulation can be performed to create knowledge. There may be privacy concerns associated

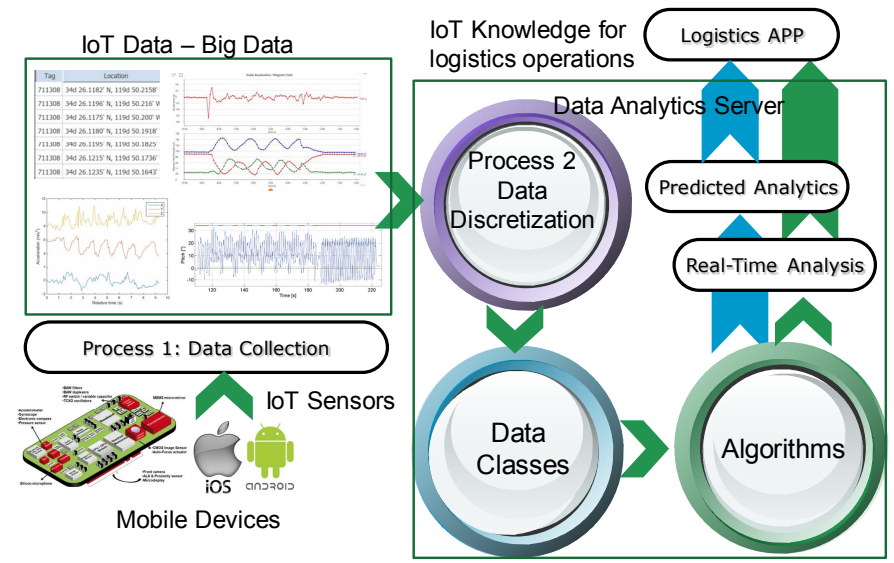

Fig. 2. Overview of the proposed system based on sensor data aquistion and manipulation.

with collecting this data, but since our proposal is targeted to professional activity monitoring this topic is not addressed in detail in this paper. Nonetheless privacy concerns may remain in terms of drivers' behavior, but that specific data can be removed from monitoring if privacy is a concern.

This collected data can be transformed into information that allows passive tracking the users' mobility activity. Therefore, we propose in this paper the development of a low cost mobile app that could assist on the distribution of urban goods in a city environment where there is a high granularity in the delivery process due the nature of the final client [6]. Passive tracking of user activities using mobile devices [7] has been assessed in a diversity of studies applied to activity recognition [8] and transportation mode detection [9], among others [10]. Through the proposed approach, our intention is to establish a common methodology to handle this collected data into a diversity of Knowledge Discovery (KD) systems, in order to facilitate the development of dedicated Intelligent Transportation System (ITS) applications.

\section{DATA ANALITICS SERVER}

After data collection (process 1), the information is transmitted to a central data analytics server, where we apply a data transformation (process 2) composed by data discretization and transformation into pre-defined class. After that, it is possible to apply data mining algorithms (e.g., Naïve Bayes, decision trees, inference along others) towards the desirable knowledge to be used by logistics apps.

Process 2 was described in detail in our previous work [2, 12]. In a first stage, we employ an outliers' identification and removal approach [12]. Then, we transform the continuous collected sensor data into predefined discrete classes. Fig. 3 illustrates some of the 24 predefined classes (C1 to C24). Classes $C 1$ to $C 4$ are based on accelerometer data on the Zaxis: $C 1$ for events where $|\Delta \alpha| \leq 0.1 \mathrm{~ms}^{-2}, C 2$ for events where $|\Delta \alpha| \epsilon(0.1,0.5] \mathrm{ms}^{-2}, C 3$ for events where $|\Delta \alpha| \epsilon(0.5,2.0] \mathrm{ms}^{-2}$ and $C 4$ for events where $|\Delta \alpha|>2 \mathrm{~ms}^{-2}$. This data can be used to identify, together with the GPS coordinates, the position of potholes and speed bumps. Classes $C 5$ to $C 8$ are based on accelerometer data on the X-axis. Fig. 3 (b) shows data from an aggressive driver, with aggressive left and right turns. C5 for 
right turn with $|\Delta \alpha| \leq 1 \mathrm{~ms}^{-2}$ and $C 6$ for left turn with $|\Delta \alpha| \leq 1 \mathrm{~ms}^{-2}, C 7$ for aggressive right turn with $|\Delta \alpha|>1 \mathrm{~ms}^{-2}$ and $C 8$ for aggressive left turn with $|\Delta \alpha|>1 \mathrm{~ms}^{-2}$. For the Y direction (Fig. 3 (c)), we divide these data events into twelve classes. $C 9$ concerns the cases when the speed is zero and $C 10$ when the speed is different from zero, which means inertial movement $\left(|\Delta \alpha|=0 \mathrm{~ms}^{-2}\right)$. Other events are used to identify moderate or aggressive acceleration and braking events: C11 for moderate acceleration $\left(|\Delta \alpha| \leq 1 \mathrm{~ms}^{-2}\right)$ and $C 12$ for moderate braking $\left(|\Delta \alpha| \leq 1 \mathrm{~ms}^{-2}\right), \quad C 13$ for excessive acceleration $\left(|\Delta \alpha|>1 \mathrm{~ms}^{-2}\right)$ and $C 14$ for excessive breaking $\left(|\Delta \alpha|>1 \mathrm{~ms}^{-2}\right)$. Speed values are taken from:

$$
V_{y}[k+1]=V_{y}[k]+t \cdot a_{y}[k],
$$

where $k$ is the elapsed time between samples and $a_{y}$ is the measurement of acceleration in the $\mathrm{Y}$-axis. The speed data is transformed into classes $C 9, C 10$, and $C 15$ to $C 20$ : $C 15$ for $V t$ $\epsilon(0,10] \mathrm{km} / \mathrm{h}, C 16$ for $V t \in(10,30] \mathrm{km} / \mathrm{h}, C 17$ for $V t \epsilon$ $(30,60] \mathrm{km} / \mathrm{h}, C 18$ for $V t \in(60,90] \mathrm{km} / \mathrm{h}, C 19$ for $V t \epsilon$ $(90,120] \mathrm{km} / \mathrm{h}$ and $C 20$ with $V t>120 \mathrm{~km} / \mathrm{h}$. Class $C 21$, for $V t<0 \mathrm{~km} / \mathrm{h}$, is used for parking operation identification. GPS data (class C24) is used to identify the route path, because we associate GPS data to route graph data available in information layers of maps, for details see [2]. The date is identified in class $C 22$ and the time, in a day time period, in class $C 23$, separated into three sub-classes: weekends and holidays, rush hours, and normal working day periods.

The algorithms referred in Fig. 2 use predefined classes and sub-classes to perform KD based on a data mining (DM) approach. Examples of this KD are driving style, road conditions, traffic, and load/unload of goods, which are implement based on a Naïve Bayes algorithm.

\section{MOBILE APP}

The developed mobile app allows to configure the sensor polling time and the type of sensors to use. One important aspect is the automatic calibration of the direction of the mobile device through the use of the magnetometer and gyroscope [13]. This process has always an error due to the change of magnetic field based on the user position. We also developed a scan tag mode, to identify goods and follow all the delivery process. This mode, together with the mobile device sensor information, allows the tracking of the urban goods and the delivery truck during all the phases of the distribution process. For example, using the mobile device motion sensors, we are able to identify user movements associated with picking and delivery of goods, such as up/down movements, use of stairs, etc.

This mobile app also uses our previous work on route optimizer algorithms and data representation on maps [14]. Fig. 4 highlights the main features available in the mobile app, including:

- Knowledge extracted from sensor data: Goods movements' follow-up process, activity statistics (number of deliveries, average delivery time, distance performed, among others), characterization of driving style, traffic information and road classification.

- Payment interface, which is not covered in this work due to space restrictions.

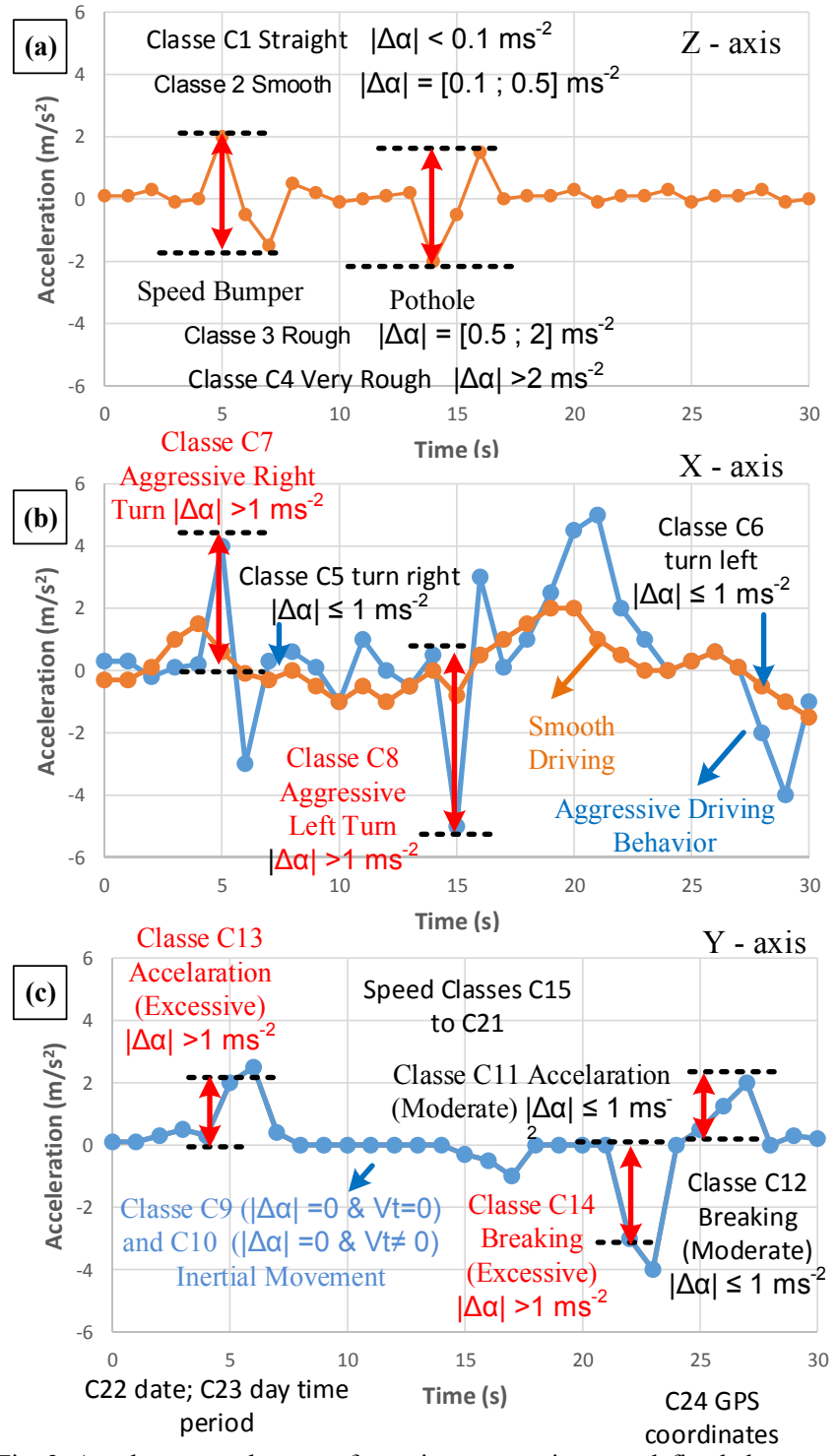

Fig. 3. Accelerometer data transformation process into pre-defined class.

- Optimization process, taking into account the available mobile device sensor data, including the optimization of performed routes based on short distance algorithms [14] and the identification of the delivery resources/processes that can be shared (see section V.D).

Other knowledge that can be extracted from the mobile device sensor data includes the information of when the deliver processes occurs, routes used, delivery operation time, road condition and driving style. It is important to compare routes and times to identify similarities in order to suggest collaboration processes among different operators.

We also store information about distance per delivery process performed in a defined period. For example, we have the following distance data for a specific week (Week 33) from an application associated to a deliver vehicle: Operator $A$ : $246 \mathrm{~km}$; Operator $B: 123 \mathrm{~km}$; and Operator $C: 81 \mathrm{~km}$. Fig. 5 shows the daily routes performed by an operator in a given day, where the route, distance and average speed are identified. We are aware that if the user turns off the data acquisition, or if the 


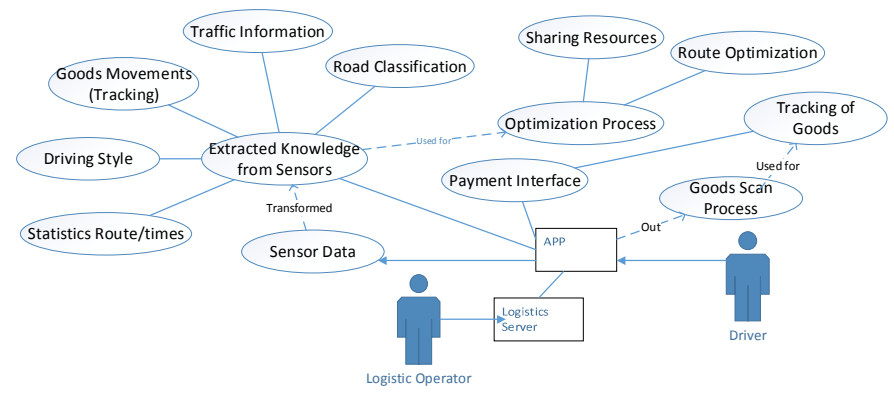

Fig. 4. Main mobile app features and outputs.

mobile device runs out of power (although the mobile device can be powered by the truck), mobility data will be lost.

\section{A. Traffic Information}

Based on the speed data and the location information provided by GPS, it is possible to identify traffic situations as conditions that occur on road networks as the number of vehicles increases, and are characterized by slower speeds and longer trip times. For this we check number of events on Classes $C 16$ to $C 20$ (speed classes) against the information about speed limit on that road. For details, see [15].

\section{B. Road Classification Application}

Accelerometer vertical measurements (Z-axis), identified as classes $C 1$ to $C 4$ (Fig 3(a)), can provide important information about road conditions. $C l$ events are small vibrations and have no meaning, $C 2$ represents rough streets with bad construction quality (bumpy roads), $C 3$ and $C 4$ represents depressions (upspeed bumpers or down potholes) and GPS shows position. Most of time, drivers can avoid potholes, which is reflected on turn left-right operations, but sometimes they cannot be avoided.

Road classification is based on events presented on classes $C 1$ to $C 4$. The main idea is to give zero points to events on class $C 1$, one point to events on class $C 2$, two points to $C 3$ and three points to class $C 4$. So we created a parameter, Road Quality (RQ), defined by equation (2), where \# means number of events on that class:

$$
R Q=(\# C 2+\# C 3 \times 2+\# C 4 \times 3) /(\# C 1+\# C 2+\# C 3+\# C 4) .
$$

If $R Q \leq 0.1$ we have a very good road, for $R Q \in(0.1,0.3]$ we have a good road, for $R Q \in(0.3,0.6]$ we have a bad road and for $R Q>0.6$ we have a very bad road. From the collected data we had 11 events classified as good road, 11 as very good road and 24 as bad road.

\section{Driving Style Characterization}

Examples of approaches in this category which use mobile devices include works carried out to determine driving behavior [16], driving style [17], and drunken behavior [18], and to evaluate driving parameters [19]. The behavior of a driver is essential in the delivery process and we can monitor this behavior taking into account speed, avoidance of road potholes, excessive breaking and acceleration events, such as in Fig. 3 (c), when the acceleration difference of two consecutive events is more than $\pm 1 \mathrm{~ms}^{-2}$, as well as aggressive change of left/right lane, such as in Fig. 3 (b), when the acceleration

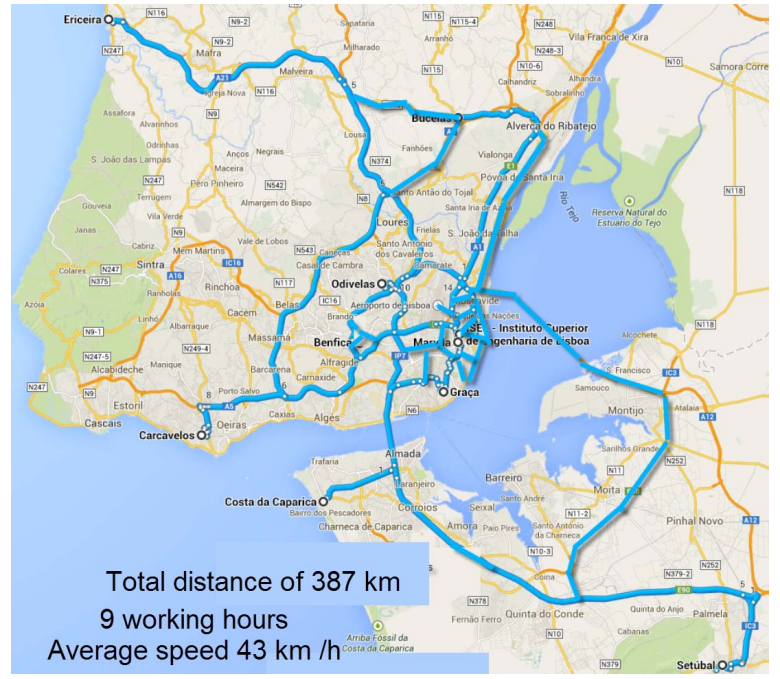

Fig. 5. Driving routes for an operator in a day period.

difference of two consecutive events is more than $\pm 1 \mathrm{~ms}^{-2}$. We applied a probabilistic approach using the number of events of change of line to left or to right, identified as turn events (TE), where \# means number of events of class:

$$
T E=\# C 5+\# C 6+\# C 7+\# C 8 \text {. }
$$

Aggressive behavior in changing lane $(C L)$ is measured with the following ratio:

$$
C L=(\# C 7+\# C 8) / T E .
$$

Then, we look at excessive acceleration ratio:

$$
E a c=\# C 13 /(\# C 13+\# C 11),
$$

and excessive breaking ratio:

$$
E b r=\# C 14 /(\# C 14+\# C 12) .
$$

Combining (4), (5) and (6) we have:

$$
D s=C L+E a c+E b r
$$

The $D s$ score based on a predefined scale allows us to classify the driving style. The number of driving style classes can be configurable, but, as a first approach, we divide in four cases:

1) Soft driving identified as $D s 1$ for $D s<0.1$;

2) Usual driving, identified as $D s 2$ for $D S \in(0.1,0.3]$;

3) Aggressive, identified as $D s 3$ for $D S \in(0.3,0.7]$;

4) Very aggressive, identified as $D s 4$ for $D S \in(0.7,1.0]$.

\section{Resouce Sharing Approach}

The sharing economy is an upcoming reality, associated to economic and social activity involving online transactions and collaboration activity towards a common goal. These processes are supported via community-based online services, with several success examples, such as Uber [20], and these processes were expanded to a diversity of activities, such as in the case of Airbnb [21], among others. Since we possess centrally stored information regarding all routes and times, it is possible in some cases to identify a goods delivery operator that performs some routes of even find some exchange points that can act as consolidation center. An example of this could use some stores or kiosks to store goods temporally in order to facilitate the sharing process. To achieve this goal, we created 
matching functions that identify carrier routes and times. First we look for route matching in a process carried out by the identification of consecutive road nodes in common, using a window time defined by the maximum time allowed by the distribution process, as exemplified in Fig. 6. We use same matching function developed for carpooling [22]. This is a heuristics process performed from the end node of the trip to the beginning with the possibility of changing goods from on transportation carrier to another one using a kiosk in the city (for details see [23]). An example is showed at Fig. 6, where, with the improved approach, operator $\mathrm{X}$ only has to perform the route from node $\mathrm{A}$ to $\mathrm{E}$. The transported goods are placed at an identified kiosk at $\mathrm{E}$ and operator $\mathrm{Z}$ picks these goods and transports them to node $\mathrm{H}$. Therefore, from nodes $\mathrm{E}$ to $\mathrm{H}$ the process was performed by only one operator $(Z)$. As result, we have eliminated the intervention of operator $\mathrm{X}$ for the distance from node $\mathrm{E}$ to $\mathrm{H}$. These synergies make sense in an urban environment, where most of deliveries have small granularity.

\section{E. Goods Picking and Delivery Process}

The accelerometer data on $\mathrm{X}$ and $\mathrm{Y}$ axes and the vehicle speed can be used to detect a parking operation based on two identified parking types used as training: longitudinal and perpendicular, which are identified in Fig. 7 and Fig. 8, respectively.

Fig. 7 starts with a stop operation $\left(V_{t}=0, C 9\right.$ class event) where we take the GPS coordinates. Then we detect a negative acceleration in $\mathrm{Y}$ direction with $C 21$ events (negative speed) and a change in direction in $\mathrm{X}$, situation identified as a $C 7$ event followed by a $C 8$ event, or the opposite way. Then, at (5) in the $\mathrm{X}$ axis we detect movement to the opposite side and the GPS position is less 2 to 3 meters (not easy to detect because GPS error associated). Then, at (6) we have again $V_{t}=0$ (C9 event).

Fig. 8 depicts a perpendicular parking. Several operations can be recognized, such as parking forward at once, characterized by small $V_{t}$ and change on $\mathrm{X}$ direction, or park backwards, characterized by $V_{t}$ small and negative and change in $\mathrm{X}$ direction. If the process is not performed at once, we have $V_{t}$ positive and negative with changes in $\mathrm{X}$ direction. If the mobile device is with the user, we can also see vertical accelerometer measurements corresponding to body movements towards picking up the goods and can recognize walking and stair climbing movements. This process requires several training examples to work without a big number of false positives.

\section{RESUlTS AND DISCUSSION}

The data collection process in the context of the proposed mobile device sensing system occurred between June $1^{\text {st }} 2016$ and Dec $31^{\text {st }} 2016$. The obtained results were based on daily trips of members of the academic community and all data was stored in a Structured Query Language (SQL) database. Driver population consisted of 20 different persons, with an average age of 39 years and 10 years of driving experience (on average), and $95 \%$ of the driver's population were male. From this data, we used 541 different registered trips with around 1400 hours.

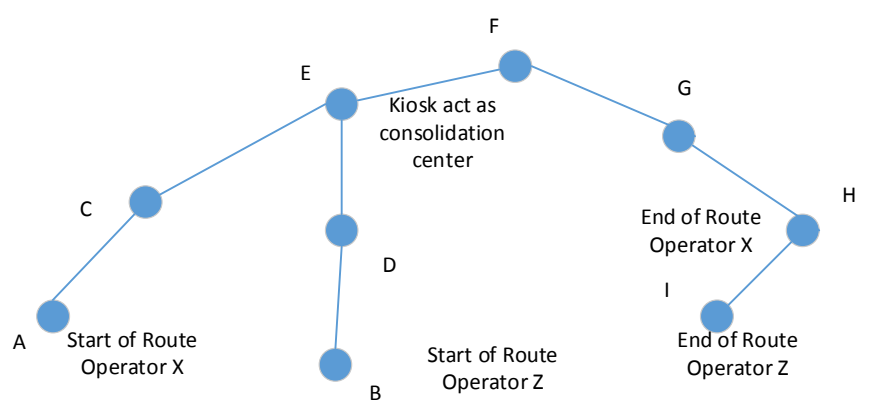

Fig. 6. Driving routes for two operators when a route matching process is performed. A comom route from node $\mathrm{E}$ to $\mathrm{H}$ is identified and performed by only one operator (in this example operator $\mathrm{Z}$ ).
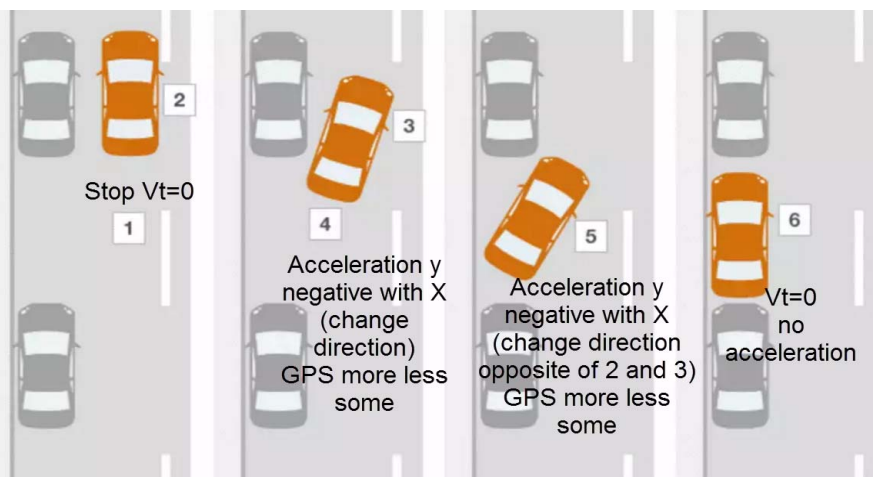

Fig. 7. Training process used to implement the identification of parking operation in a longitudinal parking parallel to the street.

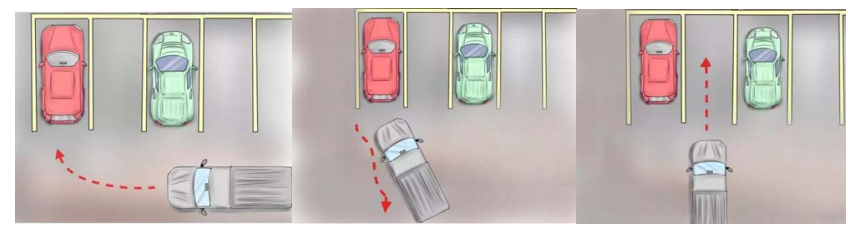

Fig. 8. Training process used to implement the identification of parking operation in a parking perpendicular to the street.

We defined two classes of braking: $\mathrm{Br}$ and $\mathrm{EBr}$ (excessive braking), when the intensity of braking is above a certain value. We highlight a few major findings: $E B r$ events per $100 \mathrm{~km}$ occur more frequently than excessive acceleration (EAc), but the number of $A c$ events tends to be higher than $\mathrm{Br}$ events per $100 \mathrm{~km}$. For example, the number of acceleration events in class $C 11$ registers values from 50 to 3400 , where $15 \%$ of these events are in the range of 50 to 510 . We can perform a normalization measurement to differentiate short to long trips $A c$ events, on average, range from 1100 to 1900 events per $100 \mathrm{~km}$. $E B r$ appears, on average, for all drivers, in the range of 100 to 130 events per $100 \mathrm{~km}$, with a maximum of 233 per $100 \mathrm{~km}$. $\mathrm{Br}$ events, on average, range from 250 to 1250 events per $100 \mathrm{~km}$, with a maximum of 3300 per $100 \mathrm{~km}$.

It was possible to identify roads with bad conditions, with several potholes identified and georeferenced. Regarding driving style, we characterized the driver population, but further research is needed to improve characterization process. Parking operation needs several heuristics to cover all possibilities of parking strategies. In this period we detected 252 parking events, where 79 where false positives (31\%) and 18 were not detected $(7 \%)$. 


\section{CONCLUSIONS}

Tracking user activity and associated mobility is available at low costs through the use of mobile device sensor information. The data generated are huge and have high impact in the study of user mobility habits. In this research we show different applications of this big data tracking using a common mining approach for knowledge discovery (KD), which can be used for specific applications, based on predefined classes. The proposed work methodology provides a bridge between field experts in data collection and data mining (DM) engineers. We also developed an IoT system based on an Android mobile application to extract sensor data and transmit it to a central server, where knowledge is extracted and can be used to characterize the goods delivery operation in a city environment. The proposed system can also be applied in rural areas.

\section{ACKNOWLEDGEMENTS}

This work has been supported by COMPETE: POCI-01-0145FEDER-007043 and FCT - Fundação para a Ciência e Tecnologia within the Project Scope: UID/CEC/00319/2013.

\section{REFERENCES}

[1] Reeves S., "Envisioning Ubiquitous Computing," ACM Annual Conference on Human Factors in Computing Systems, Austin TX USA, pp.1573-1582, May 2012.

[2] João C. Ferreira, Vítor Monteiro, José A. Afonso, João L. Afonso, "Tracking Users Mobility Patterns Towards CO2 Footprint," in Advances in Intelligent and Soft Computing, 1st ed., S. Omatu, A. Selamat, G. Bocewicz, P. Sitek, I. Nielsen, J. A. García-García, J. Bajo, Ed. AISC Springer Verlag, 2016.

[3] Weinberg $\mathrm{H}$, "Using the ADXL202 in Pedometer and Personal Navigation Applications," Analog Devices, Norwood MA USA, pp.1-8, 2002.

[4] Kim J. W., Jang H. J., Hwang D. H., Park C. A, “A Step, Stride and Heading Determination for the Pedestrian Navigation System," Journal of Global Position Systems, vol.3, no.1, pp.273-279, Dec. 2004.

[5] Scarlett J., "Enhancing the Performance of Pedometers Using a Single Accelerometer," Analog Devices, Norwood MA USA, pp.1-16, 2007.

[6] Alan Rushton, Phil Croucher, Peter Baker, "The Handbook of Logistics and Distribution Management: Understanding the Supply Chain," Edt. Kogan Page Limited, 2017.

[7] J. Jin, J. Gubbi, S. Marusic, M. Palaniswami, "An Information Framework for Creating a Smart City Through Internet of Things," IEEE Internet of Things Journal, vol.1, no.2, pp.112-121, Apr. 2014.
[8] Zhen-Yu He, Lian-Wen Jin, "Activity recognition from acceleration data using AR model representation and SVM," International Conference on Machine Learning and Cybernetics, Kunming, pp.2245-2250, July 2008.

[9] S. Reddy, M. Mun, J. Burke, D. Estrin, M. Hansen, M. Srivastava, "Using Mobile Phones to Determine Transportation Modes," ACM Trans. on Sensor Networks, vol.6, n.2, pp.13-40, Feb. 2010

[10] A. Zanella, N. Bui, A. Castellani, L. Vangelista, M. Zorzi, "Internet of Things for Smart Cities," IEEE Internet of Things Journal, vol.1, no.1, pp.22-32, Feb. 2014.

[11] Android Operating System (29 March 2017) [online]. Available online: http://www.android.com/

[12] João Ferreira, Jose Almeida, Alberto Silva, "The Impact of Driving Styles on Fuel Consumption: A Data Warehouse and Data Mining based Discovery Process," IEEE Transactions on Intelligent Transportation Systems, vol.16, no.5, pp.2653-2662, Mar. 2015.

[13] Timo Pylvänäinen, "Automatic and adaptive calibration of 3D field sensors,” ELSEVIER Applied Mathematical Modelling, vol.32, no.4, pp.575-587, Apr. 2008.

[14] João Ferreira, "Green Route Planner," Nonlinear Maps and their Applications, Selected Contributions from NOMA 2011 International Workshop. Springer Proceedings in Mathematics \& Statistics, vol.57. Eds. C. Grácio, D. F. Prunaret, T. Ueta, Y. Nishio, 2014.

[15] José Barata, Rui Ferro, João Ferreira, "My Traffic Manager," ELSEVIER Procedia Technology, vol.17, pp.209-216, 2014.

[16] H. Eren, S. Makinist, E. Akin, A. Yilmaz, "Estimating driving behavior by a smartphone," IEEE IV Intelligent Vehicles Symposium (IV), pp.234-239, June 2012.

[17] Derick A. Johnson, Mohan M. Trivedi, "Driving style recognition using a smartphone as a sensor platform," IEEE ITSC Intelligent Transportation Systems, pp.1609-1615, Oct. 2011.

[18] Jiangpeng Dai, Jin Teng, Xiaole Bai, Zhaohui Shen, Dong Xuan, "Mobile phone based drunk driving detection," IEEE International Conference on Pervasive Computing Technologies for Healthcare, pp.18, Mar, 2010.

[19] Rui Araújo, Ângela Igreja, Ricardo de Castro, Rui Esteves Araújo, "Driving coach: A smartphone application to evaluate driving efficient patterns," IEEE IV Intelligent Vehicles Symposium, pp.1005-1010, June 2012.

[20] UBER - Uber Technologies Inc. (07 April 2017) [online]. Available online: http://www.uber.com/

[21] AIRBNB - Airbnb Ireland UC (07 April 2017) [online]. Available online: http://www.airbnb.pt/

[22] João C. Ferreira, Paulo Trigo, Porfírio Filipe, "Collaborative Car Pooling System," International Conference on Sustainable Urban Transport and Environment, Paris, pp.683-687, June 2009.

[23] João C. Ferreira, Ana Lúcia Martins, Ruben Pereira, "GoodsPooling: an intelligent approach for urban logistics," International Conference on Ambient Intelligence (ISAMI'17), Porto, June 2017. 